\title{
Genomic expansion of magnetotactic bacteria reveals an early common origin of magnetotaxis with lineage-specific evolution
}

\author{
Wei Lin $\mathbb{1}^{1,2,3} \cdot$ Wensi Zhang ${ }^{1,2,3,4} \cdot$ Xiang Zhao $\mathbb{1}^{5} \cdot$ Andrew P. Roberts ${ }^{5} \cdot$ Greig A. Paterson $\mathbb{1}^{1,2,6}$. \\ Dennis A. Bazylinski ${ }^{7}$ Y Yongxin Pan ${ }^{1,2,3,4}$
}

Received: 4 October 2017 / Revised: 23 February 2018 / Accepted: 26 February 2018 / Published online: 26 March 2018

(c) The Author(s) 2018. This article is published with open access

\begin{abstract}
The origin and evolution of magnetoreception, which in diverse prokaryotes and protozoa is known as magnetotaxis and enables these microorganisms to detect Earth's magnetic field for orientation and navigation, is not well understood in evolutionary biology. The only known prokaryotes capable of sensing the geomagnetic field are magnetotactic bacteria (MTB), motile microorganisms that biomineralize intracellular, membrane-bounded magnetic single-domain crystals of either magnetite $\left(\mathrm{Fe}_{3} \mathrm{O}_{4}\right)$ or greigite $\left(\mathrm{Fe}_{3} \mathrm{~S}_{4}\right)$ called magnetosomes. Magnetosomes are responsible for magnetotaxis in MTB. Here we report the first large-scale metagenomic survey of MTB from both northern and southern hemispheres combined with 28 genomes from uncultivated MTB. These genomes expand greatly the coverage of MTB in the Proteobacteria, Nitrospirae, and Omnitrophica phyla, and provide the first genomic evidence of MTB belonging to the Zetaproteobacteria and "Candidatus Lambdaproteobacteria" classes. The gene content and organization of magnetosome gene clusters, which are physically grouped genes that encode proteins for magnetosome biosynthesis and organization, are more conserved within phylogenetically similar groups than between different taxonomic lineages. Moreover, the phylogenies of core magnetosome proteins form monophyletic clades. Together, these results suggest a common ancient origin of iron-based $\left(\mathrm{Fe}_{3} \mathrm{O}_{4}\right.$ and $\left.\mathrm{Fe}_{3} \mathrm{~S}_{4}\right)$ magnetotaxis in the domain Bacteria that underwent lineage-specific evolution, shedding new light on the origin and evolution of biomineralization and magnetotaxis, and expanding significantly the phylogenomic representation of MTB.
\end{abstract}

\section{Introduction}

Earth's global magnetic field provides a pervasive and valuable reference frame that diverse organisms use for both short- and long-distance navigation and migration. The origin

Electronic supplementary material The online version of this article (https://doi.org/10.1038/s41396-018-0098-9) contains supplementary material, which is available to authorized users.

Wei Lin

weilin0408@gmail.com

Yongxin Pan

yxpan@mail.iggcas.ac.cn

1 Key Laboratory of Earth and Planetary Physics, Institute of Geology and Geophysics, Chinese Academy of Sciences, Beijing 100029, China

2 Institutions of Earth Science, Chinese Academy of Sciences, Beijing 100029, China

3 France-China Joint Laboratory for Evolution and Development of of this navigational capability, known as magnetoreception [1] or, in prokaryotes and protozoa, magnetotaxis [2], is regarded as a major evolutionary innovation in the history of life. Magnetotactic bacteria (MTB) biomineralize intracellular, membrane-bounded, nano-sized magnetic mineral crystals of magnetite $\left(\mathrm{Fe}_{3} \mathrm{O}_{4}\right)$ and/or greigite $\left(\mathrm{Fe}_{3} \mathrm{~S}_{4}\right)$ called magnetosomes and are characterized by their ability to sense and swim along Earth's magnetic field lines [3]. Magnetosomes are the only magnetoreceptors definitively located at a

Magnetotactic Multicellular Organisms, Chinese Academy of Sciences, Beijing 100029, China

4 College of Earth Sciences, University of Chinese Academy of Sciences, Beijing 100049, China

5 Research School of Earth Sciences, Australian National University, Canberra, ACT 2601, Australia

6 Department of Earth, Ocean and Ecological Sciences, University of Liverpool, Liverpool L69 7ZE, UK

7 School of Life Sciences, University of Nevada at Las Vegas, Las Vegas, NV 89154-4004, USA 
specific site within cells so far and are a sufficiently wellcharacterized system with which the origin and evolution of magnetotaxis can be explored [4, 5].

It has previously been suggested that magnetotaxis, based on the biomineralization of $\mathrm{Fe}_{3} \mathrm{O}_{4}$ and $\mathrm{Fe}_{3} \mathrm{~S}_{4}$ in magnetosomes, in different bacterial lineages evolved independently and that MTB originated polyphyletically [6]. However, this conclusion was made prior to the identification of conserved magnetosome gene clusters (MGCs) responsible for magnetosome biomineralization and magnetotaxis in both $\mathrm{Fe}_{3} \mathrm{O}_{4^{-}}$ and $\mathrm{Fe}_{3} \mathrm{~S}_{4}$-producing $\mathrm{MTB}$, which suggests that magnetotaxis in bacteria originated only once, so that it has a monophyletic origin [7-12]. Recent phylogenetic analyses suggest an ancient origin of bacterial magnetotaxis in the Archean Eon, thereby making this behavior a primal physiological process and possibly one of the first examples of biomineralization on early Earth [13]. However, due to the patchy distribution of MTB across different phylogenetic lineages, it has been difficult to infer the evolution of magnetotaxis in prokaryotes. Whether the genes for magnetotaxis have been transferred extensively horizontally between different microorganisms or been mainly inherited through vertical transfer remains unknown [14-16].

Although MTB have been known to exist for nearly half a century, current phylogenetic information on them is based primarily on $16 \mathrm{~S}$ rRNA gene sequences, only a small fraction of which are represented by axenic cultures [15]. For a number of years, MTB were thought to phylogenetically only belong to the Alphaproteobacteria, Deltaproteobacteria, and Gammaproteobacteria classes of the Proteobacteria phylum and the Nitrospirae phylum [17-20]. Recent new evidence has revealed previously unknown, uncultured MTB to be affiliated with the candidate phylum Omnitrophica (previously known as candidate division OP3), the candidate phylum Latescibacteria (previously known as candidate division WS3), and the phylum Planctomycetes, thereby suggesting that magnetotaxis is likely more widespread in the domain Bacteria than previously thought [16, 21, 22]. Here we present metagenomic data for MTB from diverse natural environments from both the northern and the southern hemispheres. Comparison and analyses of these reconstructed genomes provide great insight into the phylogenetic diversity of MTB and the origin and evolution of magnetotaxis as well as in iron-based biomineralization on Earth.

\section{Materials and Methods}

\section{Sample collection and MTB characterization}

Surface sediments were collected from 13 locations from aquatic areas in China and Australia (Supplementary Table 1 and Supplementary Figure 1a). The collected sediments were transferred to flasks, transported to the laboratory, and incubated at room temperature without disturbance. MTB cells were enriched magnetically using a "MTB trap" [23]. The collected cells were washed and resuspended in sterile distilled $\mathrm{H}_{2} \mathrm{O}$. The morphologies of MTB cells were analyzed and characterized as described previously [24] using a JEM-2100HR transmission electron microscope operated at $200 \mathrm{kV}$, with energy dispersive spectroscopy (Oxford X-Max 80).

\section{Metagenomic sequencing, scaffold assembly, and genome binning}

Metagenomic DNA was extracted and amplified from magnetically enriched MTB cells as previously described [13]. Shotgun sequencing of metagenomic DNA from each location was performed with an Illumina HiSeq 2000 using the pairend $2 \times 125$ reads with a 600-bp insert size or using the Illumina HiSeq 4000 with the pair-end strategy of 150-bp reads with an average 270-bp insert size (Beijing Genomics Institute, Beijing, China). Illumina reads were trimmed to remove the adapter sequences and low-quality bases, and were assembled using metaSPAdes [25] with the following parameters (--only-assembler -k 31,41,51,61,71,81,91,101, 111,121). Assembled scaffolds $\geq 2500 \mathrm{bp}$ were binned separately using MetaBAT v0.26.1 [26] and MyCC [27]. Results of two binning methods for each sample were combined and a non-redundant set of bins was chosen. The acquired genomes were curated manually with two approaches: (1) using the CheckM [28] "outliers" command to identify scaffolds from bins that appear to be outliers in either GC, tetranucleotide, or coding density space relative to the expected distribution of these genomic statistics; and (2) using BLASTn or BLASTx to identify potential contaminant contigs based on their top BLAST hits. The quality and accuracy of the acquired genomes were assessed using CheckM [28] based on the taxonomic-specific workflow (domain Bacteria) and QUAST [29]. Genomes were annotated using Prokka v1.11 [30] with manual curation. Candidate magnetosome genes were checked manually and verified using the NCBI BLAST webserver [31]. The average amino-acid identity (AAI) was calculated using enveomics [32].

\section{Phylogenetic analyses}

The maximum likelihood phylogeny of genomes was constructed using RAxML v8.2.8 [33] (-m PROTGAMMAVT -f a -x 12345 -k -p 12345 -N 100) with a concatenated alignment of the conserved ubiquitous proteins identified with PhyloPhlAn [34] (Fig. 1 and Supplementary Figure 2). The VT $+\mathrm{G}$ model was used as determined by ProtTest 3.4 [35]. The genomic tree was rooted with genomes from the domain Archaea (Methanobrevibacter ruminantium and 


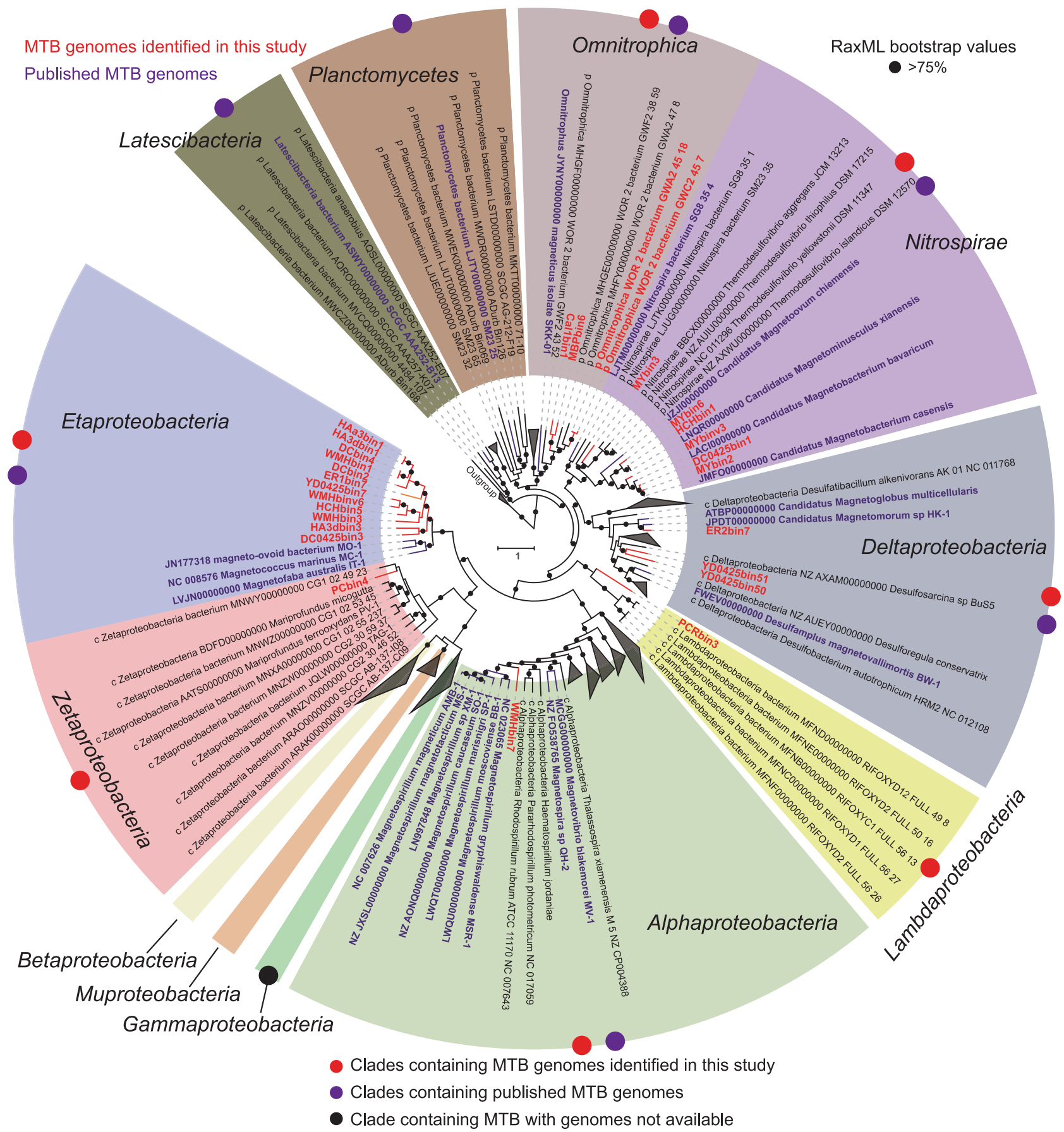

Fig. 1 Maximum likelihood phylogeny of MTB genomes. Phylogenomic tree based on concatenated alignment (3973 amino-acid positions) of up to 400 ubiquitous conserved proteins identified with PhyloPhlAn [34]. Archaeal genomes (accession numbers CP001719 and CP000678) were used as the outgroup. Red and purple denote

Methanobrevibacter smithii). Confidence in phylogenetic results was assessed using the rapid bootstrap algorithm of RAxML with 100 replicates [36]. Bootstrap convergence test was conducted using RAxML (-I autoMRE). In order to further identify whether the Magnetococcales order
MTB genomes from this study and from published MTB genome sequences, respectively. Bootstrap values are indicated with black circles (>75\% support from 100 resamples). Detailed characteristics of genome sequences recovered in this study are summarized in Supplementary Table 2 (color figure online)

represent a novel class in the Proteobacteria phylum, we additionally constructed a maximum likelihood phylogenomic tree with a concatenated amino-acid sequence alignment (6988 amino-acid positions) of 43 lineagespecific marker genes from 15 Magnetococcales genomes 


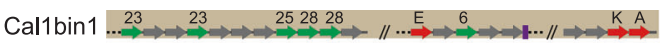

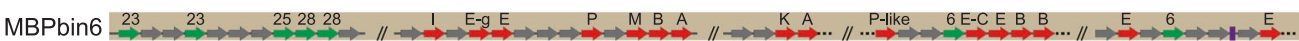

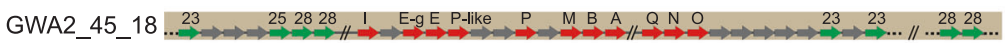

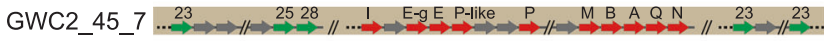

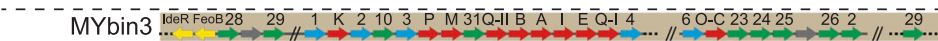

MYbin6 P. M $310-11 / \|$ B 2 A

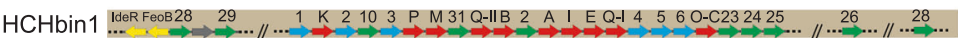

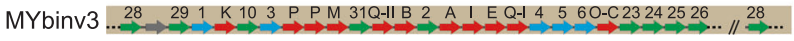

Nitrospirae

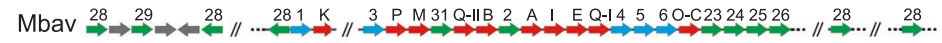

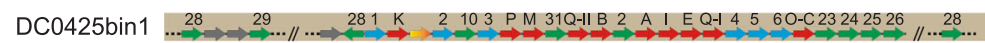

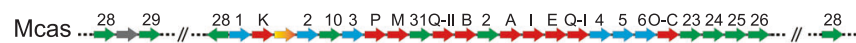

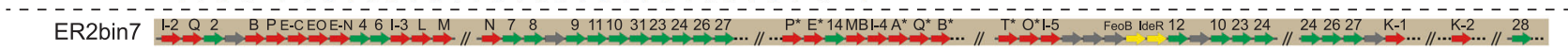

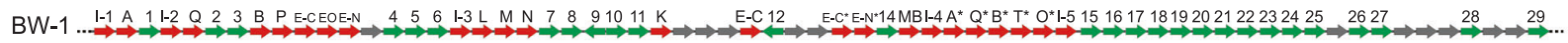

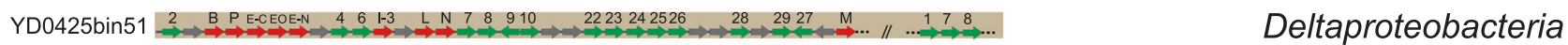

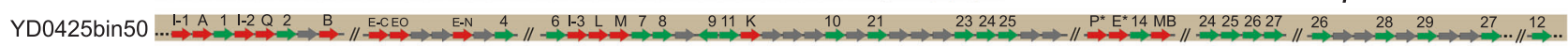

PCRbin $3 \Rightarrow$ L WMHbin7 ‥6

MSR-1 1 SF $6, G F D C$ FuA

MC-1

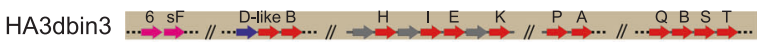

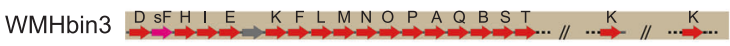

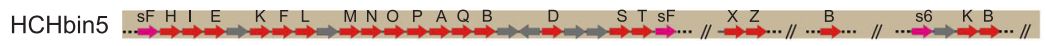

Etaproteobacteria

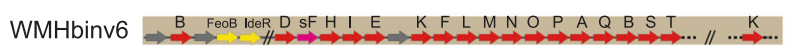

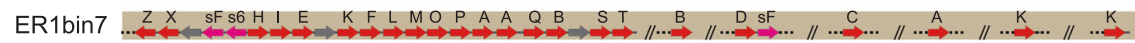

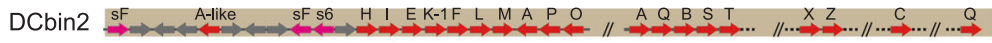

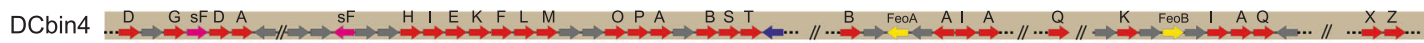

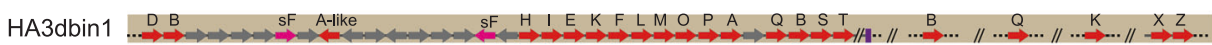

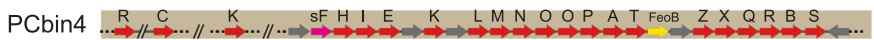

Zetaproteobacteria

mam gene $\quad m m s$ gene

man gene $\quad$ mad gene

$\square$ iron metabolism I tRNA

This study

- putative transposable element

restriction endonuclease

Fig. 2 Representative magnetosome gene clusters (MGCs) in MTB genomes. Comparison of representative MGCs recovered in this study with previously identified representative MGCs. Mbav "Candidatus Magnetobacterium bavaricum”, Mcas "Candidatus Magnetobacterium

and up to 248 Proteobacteria genomes generated using the "tree" command in CheckM [28] (Supplementary Figure 3). The genomic tree was constructed using RAxML v8.2.8 [33] under the $\mathrm{LG}+\mathrm{I}+\mathrm{G}$ model of evolution.

Homologous sequences of magnetosome proteins MamA, -B, -E, -K, -M, and -Q were identified within the refseq_protein database using PSI-BLAST searches (BLOSUM62 scoring matrix, $E$-value $<1 \mathrm{e}-05$, with exclusion of published MTB genomes) with each magnetosome protein from Magnetospirillum gryphiswaldense MSR-1, Desulfovibrio magneticus RS-1, and "Candidatus casensis", BW-1 Desulfamplus magnetovallimortis BW-1, MSR-1 Magnetospirillum gryphiswaldense MSR-1, MC-1 Magnetococcus marinus MC-1

Magnetoglobus multicellularis" as query sequences. The hits were combined and clustered using CD-HIT [37] with a sequence similarity cutoff of 0.8 . The complete amino-acid sequences of magnetosome proteins MamA, -B, -E, -K, -M, and -Q from all available MTB genomes and their nonMTB homologs were aligned by MUSCLE [38] algorithms using MEGA v6.06 [39]. Phylogenetic trees were then generated using the maximum likelihood method of FastTree v2.1.9 [40] with default settings. Multiple alignments of MamE, -M, and -Q were concatenated and a phylogenetic tree was constructed using RAxML v8.2.8 [33] with 
the $\mathrm{LG}+\mathrm{I}+\mathrm{G}$ model as determined by ProtTest 3.4 [35]. Confidence in a phylogenetic tree was assessed using 100 bootstrap replicates. Trees were visualized using FigTree v1.4.2 (http://tree.bio.ed.ac.uk/software/figtree/) and iTOL [41].

\section{Data availability}

Genome sequences have been deposited in the NCBI BioProject (Magnetotactic Bacteria Metagenome Project (MagMeta)) under accession number PRJNA400260 with genome accession numbers PDZS00000000-PEAR00000000.

\section{Results and Discussion}

\section{Metagenome-assembled MTB genomes}

To obtain a large number of MTB genomes and to better understand their genomic diversity and evolution, we conducted, to the best of our knowledge, the first large-scale metagenomic survey of MTB from varied aquatic environments, including lakes, ponds, rivers, rice fields, and creeks, in both the northern and the southern hemispheres (Supplementary Table 1 and Supplementary Figure 1a). Various MTB morphotypes, including cocci, rods, vibrios, and spirilla that contain iron-oxygen and/or iron-sulfur magnetosomes were identified in samples collected from these environments (Supplementary Figure $1 \mathrm{~b}$ and $\mathrm{c}$ ).

Metagenomic DNA sequences were extracted from magnetically enriched MTB cells and were sequenced, assembled, and binned to draft genomes. A total of 26 highquality MTB genomes were reconstructed (67-98\% completeness with $90 \%$ average; Supplementary Table 2). These new genome sequences span diverse taxonomic lineages: 18 affiliated with the phylum Proteobacteria, 6 with the phylum Nitrospirae, and 2 with the phylum Omnitrophica (Fig. 1). We also included two published genomes from the Omnitrophica phylum [42] from public databases containing nearly complete MGCs (Supplementary Table 2). These MGCs were previously unrecognized and were identified here, resulting in total 28 novel MTB genomes in this study (Fig. 1). Most of these new genomes (24 of 28) are phylogenetically divergent from previously known MTB genomes emphasizing the great phylogenetic diversity of MTB.

\section{Phylogenomic inference}

Whether classes in the Proteobacteria phylum other than the Alphaproteobacteria, Deltaproteobacteria, and Gammaproteobacteria contain MTB is a key question in understanding the evolution of magnetotaxis in this phylum
[15]. A striking finding of our study is the affiliation of two MTB genomes within the Zetaproteobacteria (PCbin4) and "Candidatus Lambdaproteobacteria" (PCRbin3) classes, respectively, in the Proteobacteria phylum (Fig. 1). The Zetaproteobacteria recognized so far are neutrophilic, lithotrophic marine $\mathrm{Fe}^{2+}$-oxidizing bacteria commonly found in $\mathrm{Fe}^{2+}$-rich environments from hydrothermal vents to coastal environments [43, 44], although members of this group have been recently identified in marine environments that do not contain elevated concentrations of $\mathrm{Fe}$ [45]. PCbin4 contains a nearly complete $\mathrm{Fe}_{3} \mathrm{O}_{4}$-type MGC (Fig. 2). Considering that all currently known isolates of Zetaproteobacteria are obligate microaerophilic, lithotrophic $\mathrm{Fe}^{2+}$-oxidizing bacteria, our results might indicate that PCbin 4 may be capable of biomineralizing both intracellular and extracellular iron minerals, although further investigation is necessary.

"Candidatus Lambdaproteobacteria" is a candidate class recently proposed based on metagenome-assembled genome sequences [42]. There are currently no cultivated representatives of this class and little is known regarding their physiology. PCRbin3 clusters robustly within the "Candidatus Lambdaproteobacteria" (100 bootstrap value) and represents the earliest diverging clade in this class (Fig. 1). The first findings of MTB from the Zetaproteobacteria and "Candidatus Lambdaproteobacteria" classes extend the phylogenetic diversity of MTB in the Proteobacteria phylum and further indicate that magnetotaxis is widespread in this phylum.

Previous cultivation-independent surveys indicate the presence of large MTB populations phylogenetically affiliated with the order Magnetococcales from both freshwater and marine habitats resulting in these organisms being considered a or the dominant MTB group in many environments [46]. Despite their widespread distribution, only three marine strains have been isolated in axenic cultures and have had their genomes sequenced, which makes the phylogenetic placement of the Magnetococcales inconclusive [47-49]. rRNA gene-based analyses indicate an affiliation of Magnetococcales within the Alphaproteobacteria class as the deepest-diverging branch [50] or even as a subclass [51]. Comparative genomic studies, however, suggest a high level of mosaic origins of Magnetococcales genomes [52] and it has been suggested recently that this order represents a new class (i.e., "Candidatus Etaproteobacteria") in the phylum Proteobacteria [47]. We reconstructed 12 Magnetococcales genomes here, which substantially expand the genomic representation of this group. To explore more accurately the phylogenetic placement of the Magnetococcales, in addition to the genomic tree constructed from concatenated conserved ubiquitous proteins using PhyloPhlAn [34] (Fig. 1 and Supplementary Figure 2), we constructed a phylogenomic tree based on a 
concatenated alignment of amino-acid sequences of marker genes identified by CheckM [28] (Supplementary Figure 3). The exact position of the Magnetococcales in two genomic trees is not consistent: in the PhyloPhlAn tree the Magnetococcales represents a sister clade to the classes of Betaproteobacteria, Gammaproteobacteria, Zetaproteobacteria, and "Candidatus Muproteobacteria" (Fig. 1), while in the CheckM tree, Magnetococcales is a sister clade to the Alphaproteobacteria (Supplementary Figure 3). Despite these inconsistencies, both trees provide strong support for Magnetococcales as a novel monophyletic class of Proteobacteria (i.e., "Candidatus Etaproteobacteria"), rather than as an order within the Alphaproteobacteria. The exact phylogenetic placement of "Candidatus Etaproteobacteria" relative to other classes in the Proteobacteria phylum awaits further investigation when more genomic sequences become available.

One (WMHbin7) and three (ER2bin7, YD0425bin50, and YD0425bin51) genomes were identified as belonging to the Alphaproteobacteria and Deltaproteobacteria classes, respectively (Fig. 1). WMHbin7 forms a distinct lineage in the order Rhodospirillales and represents a sister group to the well-characterized genus Magnetospirillum (Fig. 1). ER2bin7 is phylogenetically closely related to the uncultured, multicellular magnetotactic prokaryotes (MMPs) "Candidatus Magnetomorum" sp. HK-1 (HK-1) [53] and "Candidatus Magnetoglobus multicellularis" (MMP) [54]. The average AAI value between ER2bin7 and HK-1 is $74 \%$, which is higher than the genus criterion level of $65 \%$ [55], indicates that ER2bin7 and HK-1 belong to the same genus. Similar to HK-1, two sets of putative $\mathrm{Fe}_{3} \mathrm{O}_{4}$ and $\mathrm{Fe}_{3} \mathrm{~S}_{4}$-type magnetosome genes were identified in ER2bin7, which suggests that this organism biomineralizes both types of magnetosomes in the same types of cells (Fig. 2). Supporting this suggestion is the discovery of a population of MTB cells from sample ER2 that contain both iron-oxygen and iron-sulfur magnetosomes (Supplementary Figure 1c). The AAI value between YD0425bin50 and YD0425bin51 is only 53\%, which indicates that they represent organisms of two different genera. Both $\mathrm{Fe}_{3} \mathrm{O}_{4^{-}}$ and $\mathrm{Fe}_{3} \mathrm{~S}_{4}$-type magnetosome genes were identified in YD0425bin50, while only one MGC with high similarity to $\mathrm{Fe}_{3} \mathrm{O}_{4}$-type magnetosome genes was found in YD0425bin51 (Fig. 2). YD0425bin50 and YD0425bin51 have only a distant phylogenetic relationship to other MTB of the Deltaproteobacteria, including ER2bin7, HK-1, MMP, and Desulfamplus magnetovallimortis BW-1 (Fig. 1), which suggests that there is considerable diversity of MTB in the Deltaproteobacteria remaining to be described. Further studies, such as 16S rRNA gene-based identification and cultivation-dependent analysis, will provide a deeper insight into the diversity of magnetotactic Deltaproteobacteria.
MTB in the phylum Nitrospirae are of interest because some members of this phylum synthesize hundreds of intracellular bullet-shaped $\mathrm{Fe}_{3} \mathrm{O}_{4}$ magnetosomes and large amounts of sulfur granules [56], which suggests that they contribute significantly to iron and sulfur cycling in natural environments. We obtained six genomes that are affiliated phylogenetically with this phylum that belong clearly to two distinct clusters: one consisting of previously reported MTB of this phylum, while MYbin3, a new genus according to the low AAI values $(<60 \%)$ with respect to other genomes, is affiliated with another group that is related distantly to the genus Thermodesulfovibrio (Fig. 1). Considering that a thermophilic population of MTB distantly related to the Thermodesulfovibrio was identified from hot springs [57] and recent identification of a putative MTB genome (Nitrospira bacterium SG8_35_4) from another cluster (Fig. 1) [16], MTB of the phylum Nitrospirae are very likely more diverse than previously thought. In agreement with previous studies [12,13], the gene content and order of MGCs from the Nitrospirae were highly conserved despite the wide phylogenetic distance between organisms (Fig. 2). HCHbin1 has an average AAI value of $>99 \%$ with the genome of "Candidatus Magnetominusculus xianensis" strain $\mathrm{HCH}-1$ recovered from the same sample using a different assembly and binning approach from our previous study [13], which indicates that these two genomes are from the same organism. Similarly, the genomes of MYbin2 and "Candidatus Magnetobacterium casensis" [12] appear to be from the same organism (AAI value $>98 \%$ ).

MTB in the candidate phylum Omnitrophica were discovered only recently and so far only one population with a draft genome containing five scattered magnetosome genes has been identified [8]. Whether MTB in this phylum contain a MGC and, if so, how it is organized remains unclear. We recovered two MTB genomes (Cal1bin1 and MBPbin6) phylogenetically affiliated with this phylum. We also identified two published Omnitrophica genomes (Omnitrophica WOR_2 bacterium GWC2_45_7 and Omnitrophica WOR_2 bacterium GWA2_45_18) both containing nearly complete MGCs from the GenBank database (Fig. 2). These four genomes harbor MGCs with high similarity both in gene content and order. These MGCs, however, appear to be distinct from known MGCs from MTB of other phyla, which suggests that a hidden reservoir of MGC variants exists in previously unknown MTB lineages (Fig. 2).

\section{Characterization of MGCs}

The new genomes acquired in this study contain partial or nearly complete MGCs with genes homologous to magnetosome genes mam, mms, mad, and man, providing an opportunity to address fundamental issues concerning the 


\section{a}
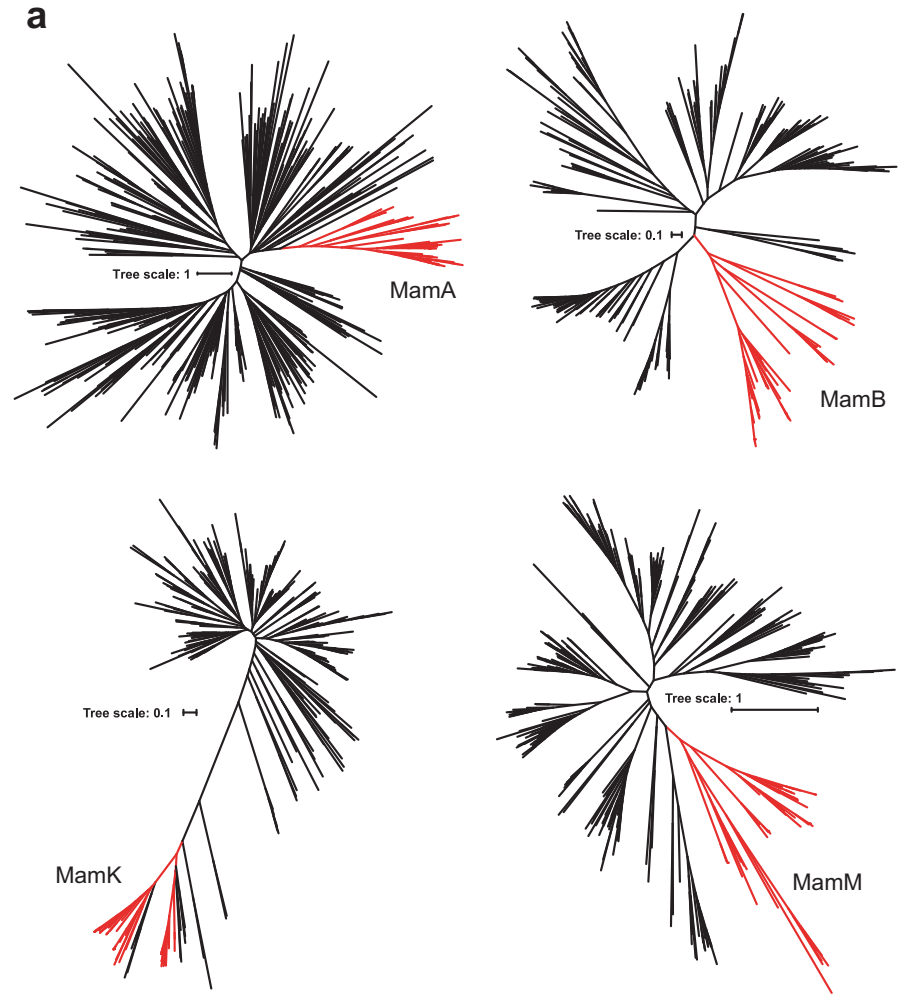
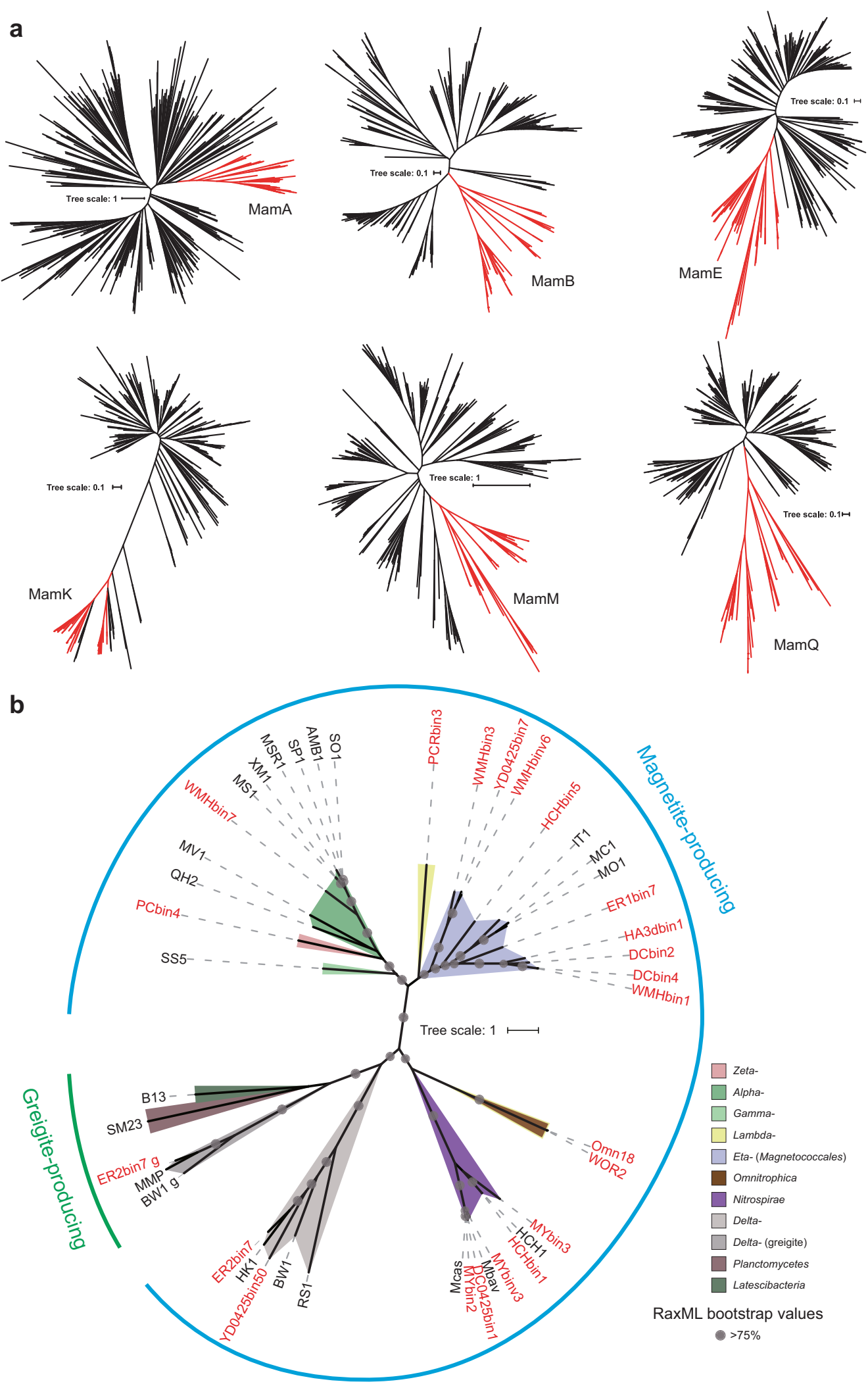

origin and evolution of magnetotaxis (Fig. 2). In general, magnetosome gene organization in each MGC corresponds with their taxonomies, that is, MGCs were more conserved in terms of gene content and organization within closely related groups than those between different taxonomic lineages (Fig. 2). The overall structures of MGCs from the 
phylum Proteobacteria (without the class Deltaproteobacteria) appear to be different from those of deepbranching MTB belonging to the class Deltaproteobacteria, the phylum Nitrospirae, and the phylum Omnitrophica. mam genes are present in all acquired MGCs, while only MGCs from deep-branching MTB contain mad and man genes. Specifically, mad genes have been identified in magnetotactic Deltaproteobacteria, Nitrospirae, and Omnitrophica, while man genes have only been found in Nitrospirae MTB (Fig. 2).

Despite general conservation, it appears that the gene order and abundance of MGCs in each class of the Proteobacteria are less conserved than those of the Nitrospirae and Omnitrophica (Fig. 2). For example, multiple copies of mamK genes have been identified within several MGCs of "Candidatus Etaproteobacteria" (e.g., WMHbin3, HCHbin5, WMHbinv6, ER1bin7, DCbin4, and HA3dbin1), but not in the other MGCs of this class, and MGCs of ER2bin7 and YD0425bin50 in the Deltaproteobacteria have considerable variability in the gene content and order (Fig. 2). One possible explanation is that this heterogeneity may be attributed to the incomplete nature of MGCs recovered through the metagenome-assembled method and/ or the artificial displaying order of scaffolds for each MGC. Conversely, this variability may represent the true nature of MGCs in these MTB. The heterogeneity of MGCs has been noted previously in axenic cultures of "Candidatus Etaproteobacteria" $[47,49,58]$ and within the magnetotactic Deltaproteobacteria [10]. Two copies of $\operatorname{mamK}$ genes have been found within MGCs of some cultivated MTB strains, such as Magnetovibrio blakemorei MV-1 and magnetotactic Gammaproteobacteria strain SS-5 [10, 11]. Genetic events, such as gene duplication, rearrangement, acquisition, and loss, may contribute to variability of MGCs, and account for the large diversity in the morphology, number, and arrangement of magnetosomes observed in MTB.

\section{Origin and evolution of magnetotaxis}

To examine the evolutionary history of magnetotaxis, amino-acid sequences of core magnetosome proteins MamA, -B, -E, -K, -M, and -Q were used to identify homologs across GenBank. For each of six protein phylogenies, magnetosome proteins all grouped together and, with the exception of MamK, the rest all form a wellsupported monophyletic clade to the exclusion of non-MTB homologs (Fig. 3a). These results support the idea that both $\mathrm{Fe}_{3} \mathrm{O}_{4^{-}}$and $\mathrm{Fe}_{3} \mathrm{~S}_{4}$-type core magnetosome genes across different taxa have a common origin. The identification of MamK, a protein involved in the construction of magnetosome chains, in the genomes of non-MTB has been previously noted [10]. It has been proposed that mamK genes in the genomes of non-MTB are either acquired through
Fig. 3 Phylogenetic analyses of core magnetosome proteins. a Phylogenies of magnetosome proteins MamA, -B, -E, -K, -M, and -Q (red) and their non-MTB homologs (black). b Maximum-likelihood phylogenetic tree based on concatenated protein alignment of MamEMQ. Sequences identified in this study are shown in red. AMB1 Magnetospirillum magneticum AMB-1, MSR-1 Magnetospirillum gryphiswaldense MSR-1, MS-1 Magnetospirillum magnetotacticum MS1, XM-1 Magnetospirillum sp. XM-1, SO-1 Magnetospirillum caucaseum SO-1, SP-1 Magnetospirillum marisnigri SP-1, MV-1 Magnetovibrio blakemorei MV-1, QH-2 Magnetospira sp. QH-2, SS-5 Gammaproteobacteria bacterium strain SS-5, IT-1 Magnetofaba australis IT-1, MC-1 Magnetococcus marinus MC-1, MO1 "Candidatus Magnetococcus massalia", HK-1 "Candidatus Magnetomorum” sp. HK-1, BW-1 Desulfamplus magnetovallimortis BW-1, RS-1 Desulfovibrio magneticus RS-1, MMP "Candidatus Magnetoglobus multicellularis", Mbav "Candidatus Magnetobacterium bavaricum", Mcas "Candidatus Magnetobacterium casensis", $\mathrm{HCH}-1$ "Candidatus Magnetominusculus xianensis" strain $\mathrm{HCH}-1$, B13 Latescibacteria bacterium SCGC AAA252-B13, SM23 Planctomycetes bacterium SM23_25 (color figure online)

horizontal gene transfer (HGT) from MTB or represent MGC relics [10]. The phylogeny based on concatenated magnetosome proteins is largely in agreement with bacterial systematics, which represents the monophyly of major classes or phyla (Fig. 3b). In addition, only a small group of acquired MGCs contain putative transposable elements and tRNA genes (Fig. 2). These results indicate that recent HGT between classes or phyla was rare during the evolution of magnetotaxis and that the origin of magnetotaxis and magnetosome biomineralization is ancient [13, 59]. The only exceptions are the $\mathrm{Fe}_{3} \mathrm{~S}_{4}$-type MGCs from the Deltaproteobacteria class, which, although distantly related, group with those from the phyla Latescibacteria and Planctomycetes (Fig. 3b).

These new data allow us to propose that the core magnetosome genes, at least for $\mathrm{Fe}_{3} \mathrm{O}_{4}$-type genes, were present in the ancestor of each of the Proteobacteria, Nitrospirae, and Omnitrophica phyla (Fig. 4a) or in the last common ancestor of the Proteobacteria, Nitrospirae, Omnitrophica, Latescibacteria, and Planctomycetes phyla (Fig. 4b). The subsequent evolutionary history of MGCs in each taxo-

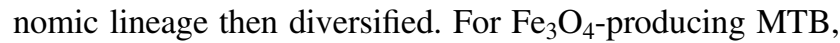
vertical inheritance followed by multiple independent losses of MGCs is likely the major force that drove the evolution of magnetotaxis, although potential recent HGT of MGCs has been suggested between some MTB within the same class or genus (e.g., Magnetospirillum) in the Proteobacteria phylum [47, 60]. Due to the limited studies of $\mathrm{Fe}_{3} \mathrm{~S}_{4}$-producing MTB, the origin and evolution of this type of MGC are poorly understood. Deltaproteobacteria MTB were the only known group containing both $\mathrm{Fe}_{3} \mathrm{O}_{4}$ - and $\mathrm{Fe}_{3} \mathrm{~S}_{4}$-type MGCs in the same genome, so it has previously been proposed that $\mathrm{Fe}_{3} \mathrm{~S}_{4}$ magnetosome formation originated in this class through duplication and subsequent 


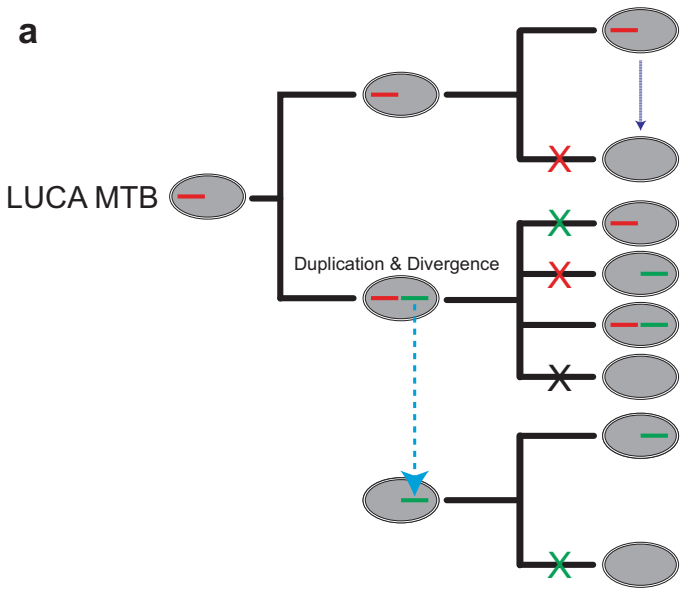

b

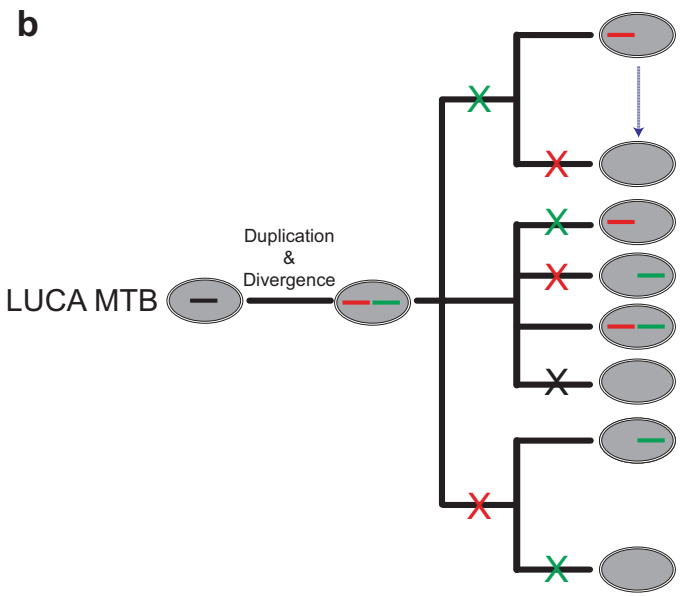

Fig. 4 Two models for the evolution of magnetotaxis in the domain Bacteria. a The last universal common ancestor of magnetotactic bacteria (LUCA MTB) was a magnetite $\left(\mathrm{Fe}_{3} \mathrm{O}_{4}\right)$-producing MTB present as the ancestor of each of the Proteobacteria, Nitrospirae, and Omnitrophica phyla. Multiple independent instances of MGC loss then ensued in each phylum or class. The $\mathrm{Fe}_{3} \mathrm{O}_{4}$-type MGC was duplicated and one diversified to a greigite $\left(\mathrm{Fe}_{3} \mathrm{~S}_{4}\right)$-type MGC in the Deltaproteobacteria. The $\mathrm{Fe}_{3} \mathrm{~S}_{4}$-type MGC is hypothesized to have been transferred to Latescibacteria and Planctomycetes. These transfer events must have occurred early because of the large phylogenetic distance between $\mathrm{Fe}_{3} \mathrm{~S}_{4}$ magnetosome proteins of Deltaproteobacteria and the Latescibacteria and Planctomycetes phyla (Fig. 3b). b In the

divergence of $\mathrm{Fe}_{3} \mathrm{O}_{4}$-type MGCs [15]. Owing to the large phylogenetic distance between magnetosome proteins of Deltaproteobacteria and of phyla Latescibacteria and Planctomycetes (Fig. 3b), we suggest that the duplication and divergence event occurred early in the Deltaproteobacteria and that MGCs in the phyla Latescibacteria and Planctomycetes were acquired through ancient HGT from the Deltaproteobacteria during evolution (Fig. 4a). Alternatively, we cannot exclude that duplication and divergence of ancient unknown MGC types generated both $\mathrm{Fe}_{3} \mathrm{O}_{4}$ - and $\mathrm{Fe}_{3} \mathrm{~S}_{4}$-type MGCs in the last common ancestor of the
Proteobacteria (without Delta-)

Nitrospirae

Omnitrophica

Delta-

\section{Latescibacteria} Planctomycetes

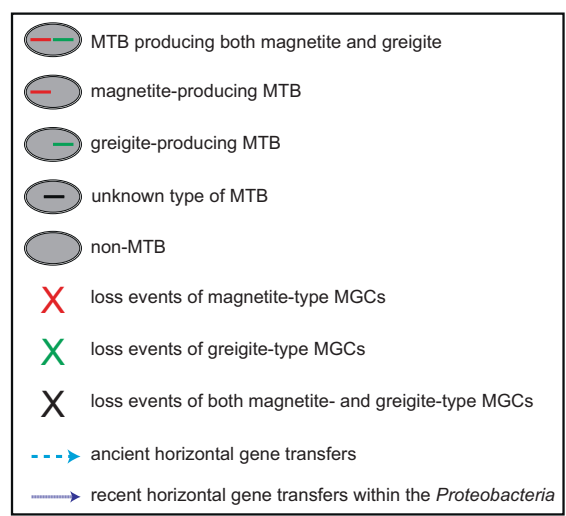

Proteobacteria (without Delta-)

Nitrospirae

Omnitrophica

Delta-

Latescibacteria

Planctomycetes

second hypothesis the LUCA MTB contained an unknown type of ancient MGC, which was duplicated and diverged to generate both $\mathrm{Fe}_{3} \mathrm{O}_{4}$ - and $\mathrm{Fe}_{3} \mathrm{~S}_{4}$-type $\mathrm{MGCs}$ in the last common ancestor of the Proteobacteria, Nitrospirae, Omnitrophica, Latescibacteria, and Planctomycetes phyla. Multiple instances of MGC loss then occurred during evolution, with some lineages losing both clusters while others retained one or two types. For both hypothetical scenarios, vertical inheritance followed by multiple independent MGC losses is considered to be the major force that drove evolution of magnetotaxis, although recent horizontal transfers of MGCs might have occurred within some classes, genera (e.g., Magnetospirillum), or species

Proteobacteria, Nitrospirae, Omnitrophica, Latescibacteria, and Planctomycetes phyla (Fig. 4b). We propose that as the phyla diversified, multiple instances of MGC loss occurred during evolution, with some lineages losing both clusters and others retaining one or both types (Fig. 4b).

The processes by which MGCs were lost multiple times across different phyla remain to be understood. The biosynthetic $\mathrm{Fe}_{3} \mathrm{O}_{4}$ magnetosome pathway is complex and has been shown to be composed of $>30$ genes and $>100 \mathrm{~kb}$ section of DNA sequence in the Magnetospirillum [3]. The frequent spontaneous loss of magnetosome genes in many 
cultivated MTB strains has been noted [61], which suggests that the metabolic cost of replicating MGCs and forming magnetosomes is high. Thus, in nature, the selective pressure driving MGC maintenance would need to be strong otherwise microorganisms would be expected to lose MGCs, thereby losing the capability of magnetosome formation when the biological advantage of magnetotaxis is small [59]. Further metagenomic and genomic sequencing of MTB from different phylogenetic lineages will undoubtedly provide valuable insights into the processes by which MTB maintained and lost MGCs.

Our work not only illustrates the unexpectedly large phylogenetic diversity of MTB in nature but also illustrates some of the possible evolutionary routes of magnetotaxis. It seems clear that magnetotaxis in the domain Bacteria is an ancient physiological trait that has a single common origin with lineage-specific evolution (i.e., it followed different evolutionary routes in different taxonomic lineages). The evolutionary history of magnetotaxis might be complex, with vertical inheritance followed by independent lineage losses as the major evolutionary force. Although the origin and evolution of $\mathrm{Fe}_{3} \mathrm{~S}_{4}$-type magnetotaxis needs further investigation, our analysis also suggests an early origin of $\mathrm{Fe}_{3} \mathrm{~S}_{4}$ magnetosomes in bacteria (Fig. 4). Detailed examination of novel MTB genomes, together with 16S rRNA gene- or magnetosome gene-based fluorescence in situ hybridization and electron microscopy studies, will further our understanding of morphological diversity, ecophysiology, and metabolic potential of these poorly characterized MTB. It should not be surprising if future work reveals new MTB with novel types of MGCs in other, as yet undiscovered, lineages across these five phyla or even across other Bacterial phyla.

Acknowledgements We thank Yuan Fang for collecting the HA3d and HAa3 samples. We thank Patrick De Deckker for suggesting field sampling sites and David Gordon and Samantha Burn for granting access to laboratory and materials at the Australian National University that enabled MTB extraction after our Australian fieldwork. WL and YP acknowledge financial support from National Natural Science Foundation of China (NSFC) grants 41330104 and 41621004. WL acknowledges support from the Youth Innovation Promotion Association of the Chinese Academy of Sciences and the Key Research Program of the Chinese Academy of Sciences grant KFZD-SW-219. APR, DAB, and YP acknowledge financial support from Australian Research Council grant DP140104544. GAP acknowledges support from NSFC grant 41574063. DAB is supported by U.S. National Science Foundation (NSF) grant EAR-1423939.

\section{Compliance with ethical standards}

Conflict of interest The authors declare that they have no conflict of interest.

Open Access This article is licensed under a Creative Commons Attribution 4.0 International License, which permits use, sharing, adaptation, distribution and reproduction in any medium or format, as long as you give appropriate credit to the original author(s) and the source, provide a link to the Creative Commons license, and indicate if changes were made. The images or other third party material in this article are included in the article's Creative Commons license, unless indicated otherwise in a credit line to the material. If material is not included in the article's Creative Commons license and your intended use is not permitted by statutory regulation or exceeds the permitted use, you will need to obtain permission directly from the copyright holder. To view a copy of this license, visit http://creativecommons. org/licenses/by/4.0/.

\section{References}

1. Winklhofer M. Magnetoreception. J R Soc Interface. 2010;7: S131-4.

2. Frankel RB, Blakemore RP. Magnetite and magnetotaxis in microorganisms. Bioelectromagnetics. 1989;10:223-37.

3. Uebe R, Schüler D. Magnetosome biogenesis in magnetotactic bacteria. Nat Rev Microbiol. 2016;14:621-37.

4. Kirschvink JL, Walker MM, Diebel CE. Magnetite-based magnetoreception. Curr Opin Neurobiol. 2001;11:462-7.

5. Benjamin LC, Jonathan TP. Identifying cellular and molecular mechanisms for magnetosensation. Annu Rev Neurosci. 2017;40:231-50.

6. DeLong EF, Frankel RB, Bazylinski DA. Multiple evolutionary origins of magnetotaxis in bacteria. Science. 1993;259:803-6.

7. Abreu F, Cantao ME, Nicolas MF, Barcellos FG, Morillo V, Almeida LG, et al. Common ancestry of iron oxide- and ironsulfide-based biomineralization in magnetotactic bacteria. ISME J. 2011;5:1634-40.

8. Kolinko S, Richter M, Glockner FO, Brachmann A, Schüler D. Single-cell genomics of uncultivated deep-branching magnetotactic bacteria reveals a conserved set of magnetosome genes. Environ Microbiol. 2016;18:21-37.

9. Lefèvre CT, Trubitsyn D, Abreu F, Kolinko S, de Almeida LGP, de Vasconcelos ATR, et al. Monophyletic origin of magnetotaxis and the first magnetosomes. Environ Microbiol. 2013;15:2267-74.

10. Lefèvre CT, Trubitsyn D, Abreu F, Kolinko S, Jogler C, de Almeida LGP, et al. Comparative genomic analysis of magnetotactic bacteria from the Deltaproteobacteria provides new insights into magnetite and greigite magnetosome genes required for magnetotaxis. Environ Microbiol. 2013;15:2712-35.

11. Lefèvre CT, Wu L-F. Evolution of the bacterial organelle responsible for magnetotaxis. Trends Microbiol. $2013 ; 21: 534-43$.

12. Lin W, Deng A, Wang Z, Li Y, Wen T, Wu L-F, et al. Genomic insights into the uncultured genus 'Candidatus Magnetobacterium' in the phylum Nitrospirae. ISME J. 2014;8:2463-77.

13. Lin W, Paterson GA, Zhu Q, Wang Y, Kopylova E, Li Y, et al. Origin of microbial biomineralization and magnetotaxis during the Archean. Proc Natl Acad Sci USA. 2017;114:2171-6.

14. Jogler C, Schüler D. Genomics, genetics, and cell biology of magnetosome formation. Annu Rev Microbiol. 2009;63:501-21.

15. Lefèvre CT, Bazylinski DA. Ecology, diversity, and evolution of magnetotactic bacteria. Microbiol Mol Biol Rev. 2013;77:497-526.

16. Lin W, Pan Y, Bazylinski DA. Diversity and ecology of and biomineralization by magnetotactic bacteria. Environ Microbiol Rep. 2017;9:345-56.

17. Sakaguchi T, Burgess JG, Matsunaga T. Magnetite formation by a sulfate-reducing bacterium. Nature. 1993;365:47-9.

18. Simmons SL, Sievert SM, Frankel RB, Bazylinski DA, Edwards KJ. Spatiotemporal distribution of marine magnetotactic bacteria in a seasonally stratified coastal salt pond. Appl Environ Microbiol. 2004;70:6230-9. 
19. Spring S, Amann R, Ludwig W, Schleifer KH, Petersen N. Phylogenetic diversity and identification of nonculturable magnetotactic bacteria. Syst Appl Microbiol. 1992;15:116-22.

20. Spring S, Amann R, Ludwig W, Schleifer KH, van Gemerden H, Petersen N. Dominating role of an unusual magnetotactic bacterium in the microaerobic zone of a freshwater sediment. Appl Environ Microbiol. 1993;59:2397-403.

21. Kolinko S, Jogler C, Katzmann E, Wanner G, Peplies J, Schüler D. Single-cell analysis reveals a novel uncultivated magnetotactic bacterium within the candidate division OP3. Environ Microbiol. 2012;14:1709-21.

22. Lin W, Pan Y. A putative greigite type magnetosome gene cluster from the candidate phylum Latescibacteria. Environ Microbiol Rep. 2015;7:237-42.

23. Jogler C, Lin W, Meyerdierks A, Kube M, Katzmann E, Flies C, et al. Towards cloning the magnetotactic metagenome: identification of magnetosome island gene clusters in uncultivated magnetotactic bacteria from different aquatic sediments. Appl Environ Microbiol. 2009;75:3972-9.

24. Lin W, Pan Y. Uncultivated magnetotactic cocci from Yuandadu park in Beijing, China. Appl Environ Microbiol. 2009;75:4046-52.

25. Nurk S, Meleshko D, Korobeynikov A, Pevzner PA. metaSPAdes: a new versatile metagenomic assembler. Genome Res. 2017;27:824-34.

26. Kang DD, Froula J, Egan R, Wang Z. MetaBAT, an efficient tool for accurately reconstructing single genomes from complex microbial communities. PeerJ. 2015;3:e1165.

27. Lin HH, Liao YC. Accurate binning of metagenomic contigs via automated clustering sequences using information of genomic signatures and marker genes. Sci Rep. 2016;6:24175.

28. Parks DH, Imelfort M, Skennerton CT, Hugenholtz P, Tyson GW. CheckM: assessing the quality of microbial genomes recovered from isolates, single cells, and metagenomes. Genome Res. 2015;25:1043-55.

29. Gurevich A, Saveliev V, Vyahhi N, Tesler G. QUAST: quality assessment tool for genome assemblies. Bioinformatics. 2013;29:1072-5.

30. Seemann T. Prokka: rapid prokaryotic genome annotation. Bioinformatics. 2014;30:2068-9.

31. Altschul SF, Madden TL, Schaffer AA, Zhang JH, Zhang Z, Miller W, et al. Gapped BLAST and PSI-BLAST: a new generation of protein database search programs. Nucleic Acids Res. 1997;25:3389-402.

32. Rodriguez-R LM, Konstantinidis KT. The enveomics collection: a toolbox for specialized analyses of microbial genomes and metagenomes. PeerJ. 2016;4:e1900v1.

33. Stamatakis A. RAxML version 8: a tool for phylogenetic analysis and post-analysis of large phylogenies. Bioinformatics. 2014;30:1312-3.

34. Segata N, Bornigen D, Morgan XC, Huttenhower C. PhyloPhlAn is a new method for improved phylogenetic and taxonomic placement of microbes. Nat Commun. 2013;4:2304.

35. Darriba D, Taboada GL, Doallo R, Posada D. ProtTest 3: fast selection of best-fit models of protein evolution. Bioinformatics. 2011;27:1164-5.

36. Stamatakis A, Hoover P, Rougemont J. A rapid bootstrap algorithm for the RAxML Web servers. Syst Biol. 2008;57:758-71.

37. Li W, Godzik A. Cd-hit: a fast program for clustering and comparing large sets of protein or nucleotide sequences. Bioinformatics. 2006;22:1658-9.

38. Edgar RC. MUSCLE: multiple sequence alignment with high accuracy and high throughput. Nucleic Acids Res. 2004;32:1792-7.

39. Tamura K, Stecher G, Peterson D, Filipski A, Kumar S. MEGA6: molecular evolutionary genetics analysis version 6.0. Mol Biol Evol. 2013;30:2725-9.
40. Price MN, Dehal PS, Arkin AP. FastTree 2-approximately maximum-likelihood trees for large alignments. PLoS ONE. 2010;5:e9490.

41. Letunic I, Bork P. Interactive tree of life (iTOL)v3: an online tool for the display and annotation of phylogenetic and other trees. Nucleic Acids Res. 2016;44:W242-5.

42. Anantharaman K, Brown CT, Hug LA, Sharon I, Castelle CJ, Probst AJ, et al. Thousands of microbial genomes shed light on interconnected biogeochemical processes in an aquifer system. Nat Commun. 2016;7:13219.

43. Field EK, Sczyrba A, Lyman AE, Harris CC, Woyke T, Stepanauskas $\mathrm{R}$, et al. Genomic insights into the uncultivated marine Zetaproteobacteria at Loihi Seamount. ISME J. 2015;9:857-70.

44. Fullerton H, Hager KW, McAllister SM, Moyer CL. Hidden diversity revealed by genome-resolved metagenomics of ironoxidizing microbial mats from Lō'ihi Seamount, Hawai'i. ISME J. 2017;11:1900-14.

45. Laufer K, Nordhoff M, Halama M, Martinez RE, Obst M, Nowak $\mathrm{M}$, et al. Microaerophilic $\mathrm{Fe}(\mathrm{II})$-oxidizing Zetaproteobacteria isolated from low-Fe marine coastal sediments: physiology and composition of their twisted stalks. Appl Environ Microbiol. 2017;83:e03118-16.

46. Lin W, Bazylinski DA, Xiao T, Wu L-F, Pan Y. Life with compass: diversity and biogeography of magnetotactic bacteria. Environ Microbiol. 2014;16:2646-58.

47. Ji B, Zhang SD, Zhang WJ, Rouy Z, Alberto F, Santini CL, et al. The chimeric nature of the genomes of marine magnetotactic coccoid-ovoid bacteria defines a novel group of Proteobacteria. Environ Microbiol. 2017;19:1103-19.

48. Araujo ACV, Morillo V, Cypriano J, Teixeira LCRS, Leão P, Lyra $\mathrm{S}$, et al. Combined genomic and structural analyses of a cultured magnetotactic bacterium reveals its niche adaptation to a dynamic environment. BMC Genomics. 2016;17:726.

49. Schübbe S, Williams TJ, Xie G, Kiss HE, Brettin TS, Martinez D, et al. Complete genome sequence of the chemolithoautotrophic marine magnetotactic coccus strain MC-1. Appl Environ Microbiol. 2009;75:4835-52.

50. Bazylinski DA, Williams TJ, Lefèvre CT, Berg RJ, Zhang CL, Bowser SS, et al. Magnetococcus marinus gen. nov., sp. nov., a marine, magnetotactic bacterium that represents a novel lineage (Magnetococcaceae fam. nov.; Magnetococcales ord. nov.) at the base of the Alphaproteobacteria. Int J Syst Evol Microbiol. 2013;63:801-8.

51. Ferla MP, Thrash JC, Giovannoni SJ, Patrick WM. New rRNA gene-based phylogenies of the Alphaproteobacteria provide perspective on major groups, mitochondrial ancestry and phylogenetic instability. PLoS ONE. 2013;8:e83383.

52. Esser C, Martin W, Dagan T. The origin of mitochondria in light of a fluid prokaryotic chromosome model. Biol Lett. 2007;3:180-4.

53. Kolinko S, Richter M, Glöckner F-O, Brachmann A, Schüler D. Single-cell genomics reveals potential for magnetite and greigite biomineralization in an uncultivated multicellular magnetotactic prokaryote. Environ Microbiol Rep. 2014;6:524-31.

54. Abreu F, Morillo V, Nascimento FF, Werneck C, Cantao ME, Ciapina LP, et al. Deciphering unusual uncultured magnetotactic multicellular prokaryotes through genomics. ISME J. 2014;8:1055-68.

55. Konstantinidis KT, Rossello-Mora R, Amann R. Uncultivated microbes in need of their own taxonomy. ISME J. 2017; 11:2399-406.

56. Jogler C, Niebler M, Lin W, Kube M, Wanner G, Kolinko S, et al. Cultivation-independent characterization of "Candidatus Magnetobacterium bavaricum" via ultrastructural, geochemical, 
ecological and metagenomic methods. Environ Microbiol. 2010;12:2466-78.

57. Lefèvre CT, Abreu F, Schmidt ML, Lins U, Frankel RB, Hedlund BP, et al. Moderately thermophilic magnetotactic bacteria from hot springs in Nevada. Appl Environ Microbiol. 2010;76:3740-3.

58. Morillo V, Abreu F, Araujo AC, de Almeida LGP, Prast AE, Farina $\mathrm{M}$, et al. Isolation, cultivation and genomic analysis of magnetosome biomineralization genes of a new genus of southseeking magnetotactic cocci within the Alphaproteobacteria. Front Microbiol. 2014;5:72.
59. Lin W, Paterson GA, Zhu Q, Wang Y, Kopylova E, Li Y, et al. Reply to Wang and Chen: an ancient origin of magnetotactic bacteria. Proc Natl Acad Sci USA. 2017;114:E5019-20.

60. Rioux J-B, Philippe N, Pereira S, Pignol D, Wu L, Ginet N. A second actin-like MamK protein in Magnetospirillum magneticum AMB-1 encoded outside the genomic magnetosome island. PLoS ONE. 2010;5:e9151.

61. Ullrich S, Kube M, Schübbe S, Reinhardt R, Schüler D. A hypervariable 130-kilobase genomic region of Magnetospirillum gryphiswaldense comprises a magnetosome island which undergoes frequent rearrangements during stationary growth. J Bacteriol. 2005;187:7176-84. 